\title{
NONLINEAR ELLIPTIC BOUNDARY VALUE PROBLEMS ${ }^{1}$
}

\author{
BY FELIX E. BROWDER
}

Communicated July 30, 1963

It is the object of the present note to present a new nonlinear version of the orthogonal projection method for proving the existence of solutions of nonlinear elliptic boundary value problems. The key point in this method is the application of a new general theorem concerning the solvability of nonlinear functional equations in a reflexive Banach space involving operators which may not be continuous. In several recent papers ([2], [3], [4], [5]) the writer obtained preliminary results in this direction involving operator equations in Hilbert space. The passage from Hilbert spaces to reflexive Banach spaces marks a tremendous increase in the power and applicability of this approach to nonlinear boundary value problems and involves a sharp development of its basic ideas.

We show the existence of variational solutions of elliptic boundary value problems for strongly elliptic systems of order $2 m$ on a domain in $R^{n}$ in generalized divergence form

$$
A u=\sum_{|\alpha| \leq m} D^{\alpha} A_{\alpha}\left(x, u, \cdots, D^{m} u\right),
$$

where the $A_{\alpha}$ are of polynomial growth in $\left(u, D u, \cdots, D^{m} u\right)$. Earlier results for equations of the form (1) were obtained in 1961-1962 by M. I. Visik ([9], [10], [11]) by a more concrete analytic approach under much stronger hypotheses than those applied in our basic existence theorem, Theorem 1 below. The result of Theorem 1 is both simpler and considerably more general than the results of Visik in the papers cited above.

Because of the potential wide applicability of our method for other nonlinear problems as well as its simplicity, we give the complete proof below.

1. Let $\Omega$ be an open subset of the Euclidean space $R^{n}$, where for convenience we assume $\Omega$ to be bounded and smoothly bounded. We denote the points of $\Omega$ by $x=\left(x_{1}, \cdots, x_{n}\right)$ and $\int f(x) d x$ denotes integration with respect to Lebesgue $n$-measure. We set

$$
D_{j}=\frac{1}{i} \frac{\partial}{\partial x_{j}} \text { for } 1 \leqq j \leqq n,
$$

\footnotetext{
1 The preparation of this paper was partially supported by NSF Grant 19751. The author is a Sloan Fellow.
} 
and for each $n$-tuple $\alpha=\left(\alpha_{1}, \cdots, \alpha_{n}\right)$, we set $D^{\alpha}=D_{1}^{\alpha_{1}} \cdots D_{n}^{\alpha_{n}}$, $|\alpha|=\sum_{j} \alpha_{j}$. By functions $u$ on $\Omega$ we shall mean $r$-vector functions $u=\left(u_{1}, \cdots, u_{r}\right)$ for a fixed positive integer $r$ where each $u_{k}$ is a complex-valued function in $\Omega$ while $D^{\alpha} u=\left(D^{\alpha} u_{1}, \cdots, D^{\alpha} u_{r}\right)$.

Let $m$ be a positive integer, $p$ a real number with $1<p<+\infty$. Then:

$$
W^{m, p}(\Omega)=\left\{u \mid u \in L^{p}(\Omega), D^{\alpha} u \in L^{p}(\Omega) \text { for }|\alpha| \leqq m\right\} .
$$

(The derivatives $D^{\alpha} u$ in this definition are taken in the sense of the theory of distributions, as they shall be below.)

$W^{m, p}(\Omega)$ is a reflexive, separable Banach space with respect to the norm

$$
\|u\|_{m, p}=\left\{\sum_{|\alpha| \leq m} \int_{\Omega}\left|D^{\alpha} u\right|^{p} d x\right\}^{1 / p} .
$$

We shall denote by $C_{c}^{\infty}(\Omega)$ the family of infinitely differentiable functions with compact support in $\Omega$, considered as a subset of $W^{m, p}(\Omega)$. Let $\langle u, v\rangle=\sum_{k} \int u_{k}(x) \tilde{v}_{k}(x) d x$ be the natural pairing between $u$ in $L^{p}(\Omega)$ and $v$ in $L^{q}(\Omega)$ with $q=p(p-1)^{-1}$.

We consider the system of differential operators

$$
A u=\sum_{|\alpha| \leq m} D^{\alpha} A_{\alpha}\left(x, u, \cdots, D^{m} u\right)
$$

where for each $\alpha, A_{\alpha}$ is an $r$-vector function of $x$ in $\Omega$, the function $u$ on $\Omega$, and all the derivatives of $u$ through order $m$.

We shall assume the following concerning $A$ :

Assumption I. The functions $A_{\alpha}$ are continuous functions of all their numerical arguments. There exists a real number $p>1$ and a continuous function $g(r)$ of the real variable $r$ such that for all $u$ in $W^{m, p}(\Omega)$, all $\alpha$ with $|\alpha| \leqq m$, and almost all $x$ in $\Omega$

$$
\begin{aligned}
& \left|A_{\alpha}\left(x, u(x), \cdots, D^{m} u(x)\right)\right| \\
& \qquad g\left(\|u\|_{\mid m, p}\right)\left\{\sum_{|\beta| \leq m}\left|D^{\beta} u(x)\right|^{p-1}+1\right\} .
\end{aligned}
$$

We may weaken (2) to the following:

$$
\begin{aligned}
& \left|A_{\alpha}\left(x, u(x), \cdots, D^{m} u(x)\right)\right| \\
& \quad \leqq g\left(\|u\|_{m, p}\right)\left\{\sum_{|\beta| \leq m}\left|D^{\beta} u(x)\right|^{(p-1)+c_{\beta \alpha}}+1\right\}
\end{aligned}
$$




$$
\begin{aligned}
\mathrm{O} \leqq C_{\beta \alpha} \leqq=\left(\frac{1}{p}-\frac{m-|\beta|}{n}\right)^{-1}\left\{(p-1) \frac{(m-|\beta|)}{n}\right. \\
\left.+\left(\frac{m-|\alpha|}{n}\right)\right\} \text { if } \frac{1}{p}-\frac{m-|\beta|}{n} \geqq 0 .
\end{aligned}
$$

To define a variational boundary value problem for the system $A$, we assume that we are given a closed subspace $V$ of $W^{m, p}(\Omega)$ with $C_{c}^{\infty}(\Omega) \subset V$, (where $p$ is the real number of Assumption I). Corresponding to the representation (1) for $A$, we have the nonlinear Dirichlet form $a(u, v)$ defined for all $u$ and $v$ in $W^{m, p}(\Omega)$ by

$$
a(u, v)=\sum_{|\alpha| \leqq m}\left\langle A_{\alpha}\left(x, u, \cdots, D^{m} u\right), D^{\alpha} v\right\rangle .
$$

Assumption I implies that $a(u, v)$ is well defined by formula (3) for all $u$ and $v$ in $W^{m, p}(\Omega)$ and that by Hölder's inequality we have

$$
|a(u, v)| \leqq g_{1}\left(\|u\|_{m, p}\right)\|v\|_{m, p}
$$

where $g_{1}(r)$ is a function of the real variable $r$ depending on the function $g$ of Assumption I.

Let $V^{*}$ be the conjugate space of $V$, i.e. the space of bounded conjugate-linear functionals on $V$. For $w \in V^{*}, v \in V$, the value of $w$ at $v$ is denoted by $(w, v)$. In particular if $f \in L^{q}(\Omega)$, the bounded conjugatelinear functional $\langle f, v\rangle$ on $V$ yields an element of $V^{*}$ which we may again denote by $f$.

We now define the variational boundary value problem corresponding to $(A, V)$ by:

Definition. Let $f \in V^{*}$. Then $u$ is said to be a solution of the variational boundary value problem for $A u=f$ corresponding to the space $V$ if $u \in V$ while for all $v$ in $V$,

$$
a(u, v)=(f, v) .
$$

If we consider the extremum problem for the integral

$$
J(u)=\int_{\Omega} F\left(x, u, \cdots, D^{m} u\right) d x
$$

with $u$ ranging over $V$, then $u$ is a critical point of the functional $J$ if and only if $u$ is a solution of the variational boundary value problem in the sense of the above definition for the given space $V$ and the system $A$ given by $A_{\alpha}=(-1)^{|\alpha|} F_{p_{\alpha}}$. Such critical points have been 
studied recently for $p=2$ by S. Smale in the context of a generalization of the Morse theory.

To formulate the hypothesis of our existence theorem, we need the following additional definition:

DEFINITION. $B$ is said to be an admissible lower order operator if

$$
B u=\sum_{|\beta| \leqq m-1} D^{\beta} B_{\beta}\left(x, u, \cdots, D^{m-1} u\right)
$$

where each $B_{\beta}$ is a continuous function of its numerical arguments and satisfies an inequality of the form

$$
\begin{aligned}
\left|B_{\beta}\left(x, u, \cdots, D^{m} u\right)\right| & \\
& \leqq g_{\beta}\left(\|u\|_{m, p}\right)\left\{\sum_{|\gamma| \leq m-1}\left|D^{\gamma} u(x)\right|^{(p-1)+C_{\beta \gamma}^{\prime}}+1\right\}
\end{aligned}
$$

where

$$
\begin{aligned}
0 \leqq c_{\beta \gamma}^{\prime}<\left(\frac{1}{p}-\frac{m-|\gamma|}{n}\right)^{-1}\left\{(p-1) \frac{(m-|\gamma|)}{n}\right. & \left.+\left(\frac{m-|\beta|}{n}\right)\right\} .
\end{aligned}
$$

THEOREM 1. Let $A$ be a system of differential operators of the form (1) satisfying Assumption I for a given value of $p, 1<p<+\infty$. Let $V$ be a closed subspace of $W^{m, p}(\Omega)$ such that $C_{c}^{\infty}(\Omega) \subset V$. Suppose that there exists an admissible lower order operator $B$ and a real-valued function $c(r)$ of the real variable $r$ with $\lim _{r \rightarrow \infty} c(r)=+\infty$ such that the following two conditions hold:

(1) If $b(u, v)$ is the nonlinear Dirichlet form corresponding to the form (6) of $B$, then for all $u$ and $v$ of $V$,

(9) $\operatorname{Re}\{a(u, u-v)-a(v, u-v)+b(u, u-v)-b(v, u-v)\} \geqq 0$.

(2) For all $u$ in $V$,

$$
\operatorname{Re}\{a(u, u)\} \geqq c\left(\|u\|_{m, p}\right)\|u\|_{m, p} .
$$

Then for every $f$ in $V^{*}$, the variational boundary problem for $A u=f$ with null V-boundary conditions has at least one solution $u$, i.e. there exists $u$ in $V$ such that $a(u, v)=(f, v)$ for all $v$ in $V$.

We deduce Theorem 1 from the following abstract theorem concerning nonlinear operators in Banach spaces.

TheOREM 2. Let $X$ be a reflexive separable Banach space, $X^{*}$ its conjugate space considered as the space of bounded conjugate linear func- 
tionals on $X$. For $w \in X^{*}, u \in X$, denote the value of $w$ at $u$ by $(w, u)$. Let $G$ be a (not necessarily linear) operator from $X$ to $X^{*}$ satisfying the following conditions:

(1) There exists a completely continuous operator $C$ from $X$ to $X^{*}$ such that for all $u$ and $v$ in $X$,

$$
\operatorname{Re}\{(G(u)-G(v), u-v)+(C(u)-C(v), u-v)\} \geqq 0 .
$$

(2) There exists a function $c(r)$ of the real variable $r$ with $c(r) \rightarrow+\infty$ as $r \rightarrow \infty$ such that for all $u$ in $X$,

$$
\operatorname{Re}(G(u), u) \geqq c(\|u\|) \cdot\|u\| .
$$

(3) $G$ is demi-continuous, i.e. it is continuous from the strong topology of $X$ to the weak topology on $X^{*}$.

Then $G$ is onto $X^{*}$, i.e. the equation $G u=w$ has a solution $u$ in $X$ for every w in $X^{*}$.

(For a special class of continuous operators, the so-called potential operators, Theorem 2 is stated by Vainberg and Kachurovski as Theorem 7 of [8]. This was pointed out to the writer by George Minty.)

Before proceeding to the proof of Theorem 1 using Theorem 2, it is interesting to give a simplified form of Theorem 2 along the lines of the Lemma of Lax-Milgram [6] for linear operators.

THEOREM 3. Let $X$ be a reflexive Banach space, $a(u, v)$ a function on $X \times X$ which is conjugate linear in $v$ but not necessarily linear in $u$. Suppose that $a(u, v)$ is separately continuous in each variable with the other held fixed. Suppose further that

(1) For $u$ and $v$ in $X$,

$$
\operatorname{Re}\{a(u, u-v)-a(v, u-v)\} \geqq 0 .
$$

(2) There exists a function $c(r)$ with $\lim _{r \rightarrow \infty} c(r)=+\infty$ such that

$$
\operatorname{Re}\{a(u, u)\} \geqq c(\|u\|) \cdot\|u\| .
$$

Then for every continuous conjugate linear functional w on $X$, there exists $u$ in $X$ such that

$$
a(u, v)=(w, v)
$$

for all $v$ in $X$.

Proof of Theorem 1 from Theorem 2. We let $X$ be the space $V$ considered with the Banach space structure induced by $W^{m, p}(\Omega)$. 
Since $W^{m, p}(\Omega)$ is reflexive and separable, $V$ is also. For $u$ and $v$ in $V$, we have already remarked that

$$
|a(u, v)| \leqq g_{1}(\|u\|)\|v\| .
$$

Hence $a(u, v)$, for fixed $u$ in $V$, is a bounded conjugate linear function of $v$ in $V$ and hence representable in the form

$$
a(u, v)=(G(u), v)
$$

where $G(u) \in V^{*}$ is uniquely determined. Furthermore, $a(u, v)=(f, v)$ for all $v$ in $V$ if and only if $G(u)=f$. Hence to prove Theorem 1 , it suffices to prove that $G$ maps $V$ onto $V^{*}$.

By a similar argument, there exists an uniquely defined operator $C$ from $X$ to $X^{*}$ such that for all $u$ and $v$ in $V$,

$$
b(u, v)=(C(u), v) .
$$

From the fact that $B$ is an admissible lower-order operator, the Sobolev imbedding theorem and Hölder's inequality, it follows that

$$
|(C u, v)| \leqq g_{2}\left(\|u\|_{m, p}\right)\|v\|_{m-1, r}
$$

where $1 / r>1 / p-1 / n$. In addition, the imbedding map J of $W^{m, p}(\Omega)$ into $W^{m-1, r}(\Omega)$ is a compact linear map.

Let $V_{1}$ be the space $V$ with the topology induced by $W^{m-1, r}(\Omega)$. Then for $u_{1}$ in $V_{1}$, there exists an unique element $C_{1} u_{1}$ of $V_{1}^{*}$ such that for every $v_{1}$ in $V_{1}, b\left(u_{1}, v_{1}\right)=\left(C_{1} u_{1}, v_{1}\right)$. Setting $u_{1}=J u, v_{1}=J v$, we see that $C=J^{*} C_{1} J$.

To apply Theorem 2 to complete the proof of Theorem 1 , it is only necessary to prove that $C$ is completely continuous and that $G$ is demi-continuous. Indeed the assumptions (1) and (2) of Theorem 2 for the particular operators $G$ and $C$ just defined are merely formal translations of conditions (1) and (2) of the hypothesis of Theorem 1. To prove that $C$ is completely continuous, it suffices to prove that $C_{1}$ is demi-continuous. For then, if $\left\{u_{k}\right\}$ is a sequence from $V$ such that $u_{k} \rightarrow u_{0}$ weakly in $V, J u_{k}$ converges to $J u_{0}$ strongly in $V_{1}$ since $J$ is compact linear and it would follow that $C_{1} J u_{k} \rightarrow C_{1} J u_{0}$ weakly in $V_{1}^{*}$. Since $J^{*}$ is compact linear (and hence completely continuous) from $V_{1}^{*}$ to $V^{*}, C u_{k}=J^{*} C_{1} J u_{k} \rightarrow J^{*} C_{1} J u_{0}=C u_{0}$ strongly in $V^{*}$.

To complete derivation of Theorem 1, it suffices to show that both $G$ and $C_{1}$ are demi-continuous. Since both operators are defined in the same way, it suffices to carry through the proof for $G$.

Lemma. $G$ is demi-continuous. 
PROOF. Let $u_{k} \rightarrow u$ strongly in $V$ as $k \rightarrow \infty$. Since $G$ maps bounded sets in $V$ into bounded sets in $V^{*}$ and the latter are precompact in the weak topology, it suffices to show that there is a subsequence of $G\left(u_{k}\right)$ converging weakly to $G(u)$ in $V^{*}$. Let $v$ be a fixed element of $V$. We may choose a subsequence of $\left\{u_{k}\right\}$ such that $u_{k}(x)$ and $D^{\alpha} u_{k}(x)$ converge almost everywhere on $\Omega$ to $u(x)$ and $D^{\alpha} u(x)$ for all $\alpha$ with $|\alpha| \leqq m$. Since $A_{\alpha}$ is continuous in all its arguments, it follows that $A_{\alpha}\left(x, u_{k}(x), \cdots, D^{m} u_{k}(x)\right)$ will converge almost everywhere on $\Omega$ to $A_{\alpha}\left(x, u(x), \cdots, D^{m} u(x)\right)$.

Let

$$
\begin{aligned}
g_{k, \alpha}(x) & =\left(1+\sum_{|\beta| \leq m}\left|D^{\beta} u_{k}(x)\right|^{(p-1)+c_{\beta \alpha}}\right), \\
g_{\alpha}(x) & =\left(1+\sum_{|\beta| \leq m}\left|D^{\beta} u(x)\right|^{(p-1)+c_{\beta \alpha}}\right) .
\end{aligned}
$$

Then $g_{k, \alpha}(x)$ will converge almost everywhere to $g_{\alpha}(x)$, and by Assumption I,

$$
h_{k, \alpha}(x)=\frac{A_{\alpha}\left(x, u_{k}, \cdots, D^{m} u_{k}\right)}{g_{k, \alpha}(x)} \rightarrow \frac{A_{\alpha}\left(x, u, \cdots, D^{m} u\right)}{g_{\alpha}(x)}=h_{\alpha}(x)
$$

boundedly a.e. in $\Omega$. On the other hand

$$
g_{k, \alpha} \rightarrow g_{\alpha} \text { as } k \rightarrow \infty
$$

in $L^{\alpha}(\Omega)$ where $q=p(p-1)^{-1}$. Hence, since $\bar{h}_{k, \alpha} D^{\alpha} v$ for $|\alpha| \leqq m$ will converge strongly to $\bar{h}_{\alpha} D^{\alpha} v$ in $L^{p}(\Omega)$ by the Lebesgue dominated convergence theorem

$$
\begin{aligned}
\left(G\left(u_{k}\right), v\right) & =a\left(u_{k}, v\right)=\sum_{|\alpha| \leq m}\left\langle A_{\alpha}\left(x, u_{k}, \cdots, D^{m} u_{k}\right), D^{\alpha} v\right\rangle \\
& =\sum_{|\alpha| \leq m}\left\langle g_{k, \alpha}, \bar{h}_{k, \alpha} D^{\alpha} v\right\rangle \\
& \rightarrow \sum_{|\alpha| \leq m}\left\langle g_{\alpha}, \bar{h}_{\alpha} D^{\alpha} v\right\rangle \\
& =\sum_{|\alpha| \leqq m}\left\langle A_{\alpha}\left(x, u, \cdots, D^{m} u\right), D^{\alpha} v\right\rangle \\
& =a(u, v)=(G(u), v) .
\end{aligned}
$$

Thus for this subsequence, $G\left(u_{k}\right)$ converges weakly to $G(u)$, the Lemma is proved, and the deduction of Theorem 1 from Theorem 2 is complete.

2. Let $X$ be a complex Banach space which we assume to be separa- 
ble and reflexive, $X^{*}$ its conjugate space, i.e. the space of bounded conjugate-linear functionals on $X$. For $w \in X^{*}, u \in X$, the value of $w$ at $u$ is denoted by $(w, u)$.

Let $G$ be a mapping from an open set $D$ of $X$ into $X^{*}$.

Definition. $G$ is said to be demi-continuous if $G$ is continuous from the strong topology on $D$ to the weak topology on $X^{*}$.

Lemma 1. Let $G$ be a demi-continuous mapping of the open set $D$ of $X$ into $X^{*}$. Suppose that for fixed $u_{0}$ in $D$ and $w$ in $X^{*}$,

$$
\operatorname{Re}\left(w-G(u), u_{0}-u\right) \geqq 0
$$

for all $u$ in a dense subset $Y$ of $D$.

Then $w=G\left(u_{0}\right)$.

Proof of Lemma 1. Let $u$ be an arbitrary element of $D$. Since $Y$ is dense in $D$, there exists a sequence $\left\{v_{k}\right\}$ from $Y$ such that $v_{k}$ converges strongly to $u$ in $D$ as $k \rightarrow \infty$. Since $G$ is demi-continuous, $G\left(v_{k}\right)$ converges weakly to $G(u)$ in $X^{*}$. For each $k$, the inequality (2.1) holds with $u$ replaced by $v_{k}$, i.e.

$$
\operatorname{Re}\left(w-G\left(v_{k}\right), u_{0}-v_{k}\right) \geqq 0 .
$$

However, $w-G\left(v_{k}\right) \rightarrow w-G(u)$ weakly in $X^{*}$ while $u_{0}-v_{k}$ converges strongly to $u_{0}-u$, as $k \rightarrow \infty$. Therefore

$$
\left(w-G\left(v_{k}\right), u_{0}-v_{k}\right) \rightarrow\left(w-G(u), u_{0}-u\right) .
$$

Therefore

$$
\operatorname{Re}\left(w-G(u), u_{0}-u\right) \geqq 0,
$$

is true for all $u$ in $D$.

We assume that $w \neq G\left(u_{0}\right)$ and prove a contradiction. Under this assumption, there exists $v$ in $X$ such that

$$
\left(w-G\left(u_{0}\right), v\right)>0 .
$$

For $t>0$ and sufficiently small, $u_{t}=u_{0}+t v$ lies in $D$. Applying the inequality (2.2) with $u$ replaced by $u_{t}$, we obtain

$$
0 \leqq \operatorname{Re}\left(w-G\left(u_{t}\right), u_{0}-u_{t}\right)=\operatorname{Re}\left(G\left(u_{t}\right)-w, t v\right) .
$$

Cancelling the positive multiplier $t$, we get

$$
\operatorname{Re}\left(G\left(v_{t}\right)-w, v\right) \geqq 0,
$$

or, by an obvious calculation,

$$
\operatorname{Re}\left(G\left(u_{t}\right)-G\left(u_{0}\right), v\right) \geqq \operatorname{Re}\left(w-G\left(u_{0}\right), v\right) .
$$


As $t \rightarrow 0$, the left-hand side of the last inequality converges to zero by the demi-continuity of $G$. The right-hand side is independent of $t$. Hence

$$
0 \geqq \operatorname{Re}\left(w-G\left(u_{0}\right), v\right)>0,
$$

which is a contradiction proving the Lemma.

Definition. $G$ is said to be monotone if for all $u$ and $v$ of $D$

$$
\operatorname{Re}(G(u)-G(v), u-v) \geqq 0 .
$$

(For Hilbert spaces, this definition is due to Minty [7]).

Definition. The mapping $C$ of $X$ into $X^{*}$ is said to be completely continuous if $C$ is continuous from the weak topology on $X$ to the strong on $X^{*}$.

Definition. $P$ is said to be a projection of $X$ onto the closed subspace $F$ if $P$ is a bounded linear operator of $X$ whose range is $F$ with $P^{2}=P$. $A$ sequence of projections $\left\{P_{j}\right\}$ is said to be commutative increasing if for $j<k, P_{j} P_{k}=P_{k} P_{j}=P_{j}$.

Lemma 2. Let $\left\{F_{j}, j=1,2, \cdots\right\}$ be a sequence of finite dimensional subspaces of $X$ with $F_{j} \subset F_{j+1}$ for all $j$. Suppose $P_{1}$ is a projection of $X$ onto $F_{1}$. Then:

(a) There exists a commutative increasing family of projections $\left\{P_{j}\right\}$ beginning with $P_{1}$ and such that $F_{j}$ is the range of $P_{j}$.

(b) If $F_{j}^{\prime}$ is the range of $P_{j}^{*}$, then $\left\{P_{j}^{*}\right\}$ is a commutative increasing family of projections on $F_{j}^{\prime}$ and $F_{j}^{\prime} \subset F_{g+1}^{\prime}$ for every $j$. The pairing (w, $u$ ) for $w \in F_{j}^{\prime}$ and $u \in F_{j}$ yields an isomorphism of $F_{j}^{\prime}$ with $F_{j}^{*}$.

Proof of Lemma 2 (a). $P_{1}$ is given. We construct the projections $P_{r}$ by recursion assuming at the $r$ th step that

$$
P_{j} P_{k}=P_{k} P_{j}=P_{j}, \quad j<k \leqq r .
$$

We may assume without loss of generality that the codimension of $F_{j}$ in $F_{j+1}$ is one. Suppose we have constructed $\left\{P_{1}, \cdots, P_{r}\right\}$ satisfying the equations of (2.5) and wish to construct $P_{r+1}$ so that

$$
P_{j} P_{k}=P_{k} P_{j}=P_{j}, \quad j<k \leqq r+1 .
$$

For $k \leqq r,(2.6)$ follows from (2.5). Since for $j<k, F_{j} \subset F_{k}$, it is always true that $P_{k} P_{j}=P_{j}$ if $P_{j}$ and $P_{k}$ are projections on $F_{j}$ and $F_{k}$ respectively with $j<k$. In order that $P_{j} P_{r+1}=P_{j}$, on the other hand, it suffices that $P_{r} P_{r+1}=P_{r}$, since then

$$
P_{j} P_{r+1}=\left(P_{j} P_{r}\right) P_{r+1}=P_{j} P_{r} P_{r+1}=P_{j} P_{r}=P_{r} \text { for } j<r .
$$

The nullspace of $P_{r}$ restricted to $F_{r+1}$ is of dimension 1 and gener- 
ated by $u_{0} \neq 0$. By the Hahn-Banach theorem, there exists $w$ in $X^{*}$ such that $\left(w, u_{0}\right)=1$ while $(w, u)=0$ for all $u$ in $F_{r}$. We set $P_{r+1} u$ $=(u, w) u_{0}+P_{r} u$. Obviously $P_{r+1} u_{0}=u_{0}$, while for $u$ in $F_{r}, P_{r+1} u=P_{r} u$ $=u$. Thus $P_{r+1} u=u$ for all $u$ in $F_{r+1}$. Moreover, the range of $P_{r+1}$ is contained in $F_{r+1}$. Hence $P_{r+1}$ is a bounded projection of $X$ on $F_{r+1}$. Furthermore, $P_{r} P_{r+1} u=P_{r}\left\{(u, w) u_{0}+P_{r} u\right\}=P_{r}^{2} u=P_{r} u$, so that $P_{r} P_{r+1}=P_{r}$ and the $(r+1)$ st step of the recursion is complete.

Proof of Lemma 2 (b). Since $P_{j}^{2}=P_{j}$ and $P_{j} P_{k}=P_{k} P_{j}=P_{j}$ for $j<k$, we find by taking adjoints that

$$
\left(P_{j}^{*}\right)^{2}=\left(P_{j}^{2}\right)^{*}=P_{j}^{*}, P_{j}^{*} P_{k}^{*}=P_{k}^{*} P_{j}^{*}=P_{j}^{*}, \text { for } j<k .
$$

Hence $\left\{P_{j}^{*}\right\}$ is a commutative increasing family of projections of $X^{*}$ with $F_{j}^{\prime}=$ range of $P_{j}^{*}$. Since for $u \in F_{j}^{\prime}, P_{k}^{*} u=P_{k}^{*} P_{j}^{*} u=P_{j}^{*} u=u$ for every $k>j$, it follows that $F_{j}^{\prime} \subset F_{k}^{\prime}$ for $j<k$.

Let $K_{j}$ be the linear mapping of $F_{j}^{\prime}$ into $F_{j}^{*}$ defined by $\left(K_{j} w, u\right)$ $=(w, u)$ for all $u \in F_{j} . K_{j}$ is one-to-one since $K_{j} w=0$ implies that $\left(w, P_{j} u\right)=0$ for all $u \in X$, or $P_{j}^{*} w=0$. Since $P_{j}^{*} w=w$ for $w \in F_{j}^{\prime}$, it follows that $w=0 . K_{j}$ is also onto since for $w_{1} \in F_{j}^{*}$, there exists $w_{2}$ in $X^{*}$ so that $\left(w_{2}, u\right)=\left(w_{1}, u\right)$ for all $u$ in $F_{j}$ while $\left\|w_{1}\right\|=\left\|w_{2}\right\|$. However, $\left(K_{j} P_{j}^{*} w_{2}, u\right)=\left(P_{j}^{*} w_{2}, u\right)=\left(w_{2}, u\right)=\left(w_{1}, u\right)$ for $u$ in $F_{j}$, so that $K_{j}\left(P_{j}^{*} w_{2}\right)$ $=w_{1}$. We know finally, that $\left\|K_{j} w\right\| \leqq\|w\|$ since $K_{j} w$ is the restriction of $w$ to a subspace. Hence the proof of the Lemma is complete.

Lemma 3. Let $G$ be a demi-continuous map of $X$ into $X^{*}, G=G_{0}+C_{1}$, $G_{0}$ monotone, $C$ completely continuous. Let $\left\{F_{j}\right\}$ be an increasing sequence of finite-dimensional subspaces of $X$ whose union is dense in $X$, $\left\{P_{j}\right\}$ a commutative increasing family of projections of $X$, with $F_{j}$ $=$ range $P_{j}$. Let $\left\{u_{k}\right\}$ be an infinite sequence in $X$ such that $u_{k} \in F_{k}$ for each $k, u_{k}$ converges weakly to $u_{0}$ in $X$ as $k \rightarrow \infty$, and $P_{k}^{*} G\left(u_{k}\right)$ converges strongly to $w$ in $X^{*}$.

Then $w=G\left(u_{0}\right)$.

Proof of Lemma 3. Let $j$ be a fixed integer, $u$ an element of $F_{j}$ so that $P_{j} u=u$. Using the monotonicity inequality, we have for each $k$

$$
\operatorname{Re}\left(u_{k}-P_{j} u, G_{0}\left(u_{k}\right)-G_{0}\left(P_{j} u\right)\right) \geqq 0 .
$$

Since $u_{k}$ converges weakly to $u_{0}$, it follows that

$$
\operatorname{Re}\left(u_{k}-P_{j} u, G_{0}\left(P_{j} u\right)\right) \rightarrow\left(u_{0}-P_{j} u, G_{0}\left(P_{j} u\right)\right)
$$


as $k \rightarrow \infty$. Since $u_{k} \in F_{k}, P_{k} u_{k}=u_{k}$. For $k>j, P_{k} P_{j}=P_{j}$. Hence

$$
\begin{aligned}
\operatorname{Re}\left(u_{k}-P_{j} u, G_{0}\left(u_{k}\right)\right) & =\operatorname{Re}\left(P_{k} u_{k}-P_{k} P_{j} u, G\left(u_{k}\right)\right)-\operatorname{Re}\left(u_{k}-P_{j} u, C\left(u_{k}\right)\right) \\
& =\operatorname{Re}\left(u_{k}-P_{j} u, P_{k}^{*} G\left(u_{k}\right)\right)-\operatorname{Re}\left(u_{k}-P_{j} u, C\left(u_{k}\right)\right) .
\end{aligned}
$$

Since as $k \rightarrow \infty \quad u_{k}$ converges weakly to $u_{0}$ in $X$ and $C$ is completely continuous, $C\left(u_{k}\right)$ converges strongly to $C\left(u_{0}\right)$. Hence

$$
\operatorname{Re}\left(u_{k}-P_{j} u, C\left(u_{k}\right)\right) \rightarrow \operatorname{Re}\left(u_{0}-P_{j} u, C\left(u_{0}\right)\right)
$$

as $k \rightarrow \infty$. Finally, since $P_{k}^{*} G\left(u_{k}\right)$ converges strongly to $w$, we have as $k \rightarrow \infty$,

$$
\operatorname{Re}\left(u_{k}-P_{j} u, P_{k}^{*} G\left(u_{k}\right)\right) \rightarrow \operatorname{Re}\left(u_{0}-P_{j} u, w\right) .
$$

Collecting the various limits, we have

$$
\operatorname{Re}\left(u_{0}-u,\left\{w-C\left(u_{0}\right)\right\}-G_{0}(u)\right) \geqq 0
$$

for all $u$ in $F_{j}$. Since $j$ is arbitrary, the inequality (2.7) is true for all $u$ in $U_{j} F_{j}$, a dense subset of $X$. Applying Lemma 1 , we see that $w-C\left(u_{0}\right)=G_{0}\left(u_{0}\right)$, i.e. $w=G\left(u_{0}\right)$. Q.E.D.

LEMMA 4. Let $G$ be a continuous mapping of the finite dimensional Banach space $Y$ into $Y^{*}$ such that

$$
\operatorname{Re}(G(u), u) \geqq c(\|u\|) \cdot\|u\|
$$

where $c(r) \rightarrow+\infty$ as $r \rightarrow \infty$. Then $G$ is onto.

Proof of Lemma 4. Since $Y$ is finite dimensional, there exists a bicontinuous linear map $J$ of a Hilbert space $H$ onto $Y$. Let $J^{*}$ be the dual map of $Y^{*}$ onto $H=H^{*}$. Consider $G^{\prime}=J^{*} G J$. Then for $L \in H$

$$
\begin{aligned}
\operatorname{Re}\left(G^{\prime} h, h\right)=\operatorname{Re}\left(J^{*} G J h, h\right)=\operatorname{Re}(G J h, J h) & \geqq c(\|J h\|)\|J h\| \\
& \geqq c_{1}(\|h\|)\|h\| .
\end{aligned}
$$

Thus it suffices to consider the map $G^{\prime}$ of the Hilbert space $H$ into itself. We deform $G^{\prime}$ into the identity through the family $G_{i}^{\prime}=t G^{\prime}$ $+(1-t) I$. Let $w \in H$. For $u$ in $H, 0 \leqq t \leqq 1$

$$
\operatorname{Re}\left(G_{t}^{\prime}(u)-w, u\right)=t \operatorname{Re}\left(G^{\prime}(u), u\right)+(1-t)\|u\|^{2}-\|w\| \cdot\|u\| \geqq \frac{1}{2}\|u\|
$$

for $\|u\| \geqq M$, so that $G_{t}^{\prime}(u) \neq w$. Hence the degree of the map $G_{t}^{\prime}$ on the ball $\|u\|<M$ with respect to $w$ is independent of $t$ and for $t=0$, it is different from 0 . Hence there exists $u$ with $\|u\|<M$ and $G^{\prime}(u)$ $=w$. Thus $G^{\prime}$ is onto and so is $G$. (This argument is essentially due to Visik [9].) 
Proof of Theorem 2. Let $w$ be a fixed element of $X^{*}$. We shall show that $w$ lies in the range of $G$. Let $F_{1}^{\prime}$ be the one-dimensional subspace of $X^{*}$ spanned by $w, P_{1}^{\prime}$ a bounded projection of $X^{*}$ onto $F_{1}^{\prime}$. Let $P_{1}=\left(P_{1}^{\prime}\right)^{*}$ and let $F_{1}$ be the range of the projection $P_{1}$. By the separability of $X$, starting with $F_{1}$ we may construct an increasing sequence $\left\{F_{j}\right\}$ of finite dimensional subspaces of $X$ so that $U_{j} F_{j}$ is dense in $X$. By Lemma 2 , we may construct a commuting increasing sequence of projections $\left\{P_{j}\right\}$ of $X$ starting with $P_{1}$ and such that $F_{j}=$ range $P_{j}$.

For each fixed $k$, let $G_{k}$ be the mapping of $F_{k}$ into $F_{k}^{\prime}$ given by

$$
G_{k} u=P_{k}^{*} G u, u \in F_{k} \text {. }
$$

For $u \in F_{k}$

$$
\operatorname{Re}\left(G_{k} u, u\right)=\operatorname{Re}\left(P_{k}^{*} G u, u\right)=\operatorname{Re}\left(G u, P_{k} u\right)=\operatorname{Re}(G u, u) \geqq c(\|u\|) \cdot\|u\|,
$$

while by the demi-continuity of $G$ and the finite dimensionality of $F_{k}^{\prime}, G_{k}$ is continuous from $F_{k}$ to $F_{k}^{\prime}$. Since $F_{k}^{\prime}$ is canonically isomorphic with $F_{k}^{*}$, it follows from Lemma 4 that $G_{k}$ is onto. Hence there exists $u_{k}$ in $F_{k}$ with

$$
G_{k} u_{k}=P_{k}^{*} G u_{k}=w
$$

since $w$ lies in $F_{1}^{\prime} \subset F_{k}^{\prime}$ for all $k \geqq 1$.

Taking the real part of the inner product of equation (2.8) with $u_{k}$, we obtain

$$
\left(w, u_{k}\right)=\operatorname{Re}\left(P_{k}^{*} G u_{k}, u_{k}\right)=\operatorname{Re}\left(G u_{k}, u_{k}\right) \geqq c\left(\left\|u_{k}\right\|\right)\left\|u_{k}\right\| .
$$

Hence

$$
c\left(\left\|u_{k}\right\|\right) \leqq\|w\|
$$

implying that $\left\|u_{k}\right\| \leqq M_{1}$ since $c(r) \rightarrow+\infty$ as $r \rightarrow+\infty$. Since $X$ is reflexive, we may choose a weakly convergent subsequence of the $\left\{u_{k}\right\}$ which we can assume to be the original sequence. Then $u_{k} \rightarrow u_{0}$ weakly in $X, P_{k}^{*} G\left(u_{k}\right)=w$ converges strongly to $w$ in $X^{*}$. Hence by Lemma 3 , $G\left(u_{0}\right)=w$, and the proof of the Theorem is complete.

Proof of Theorem 3. Let $G$ be the operator from $X$ to $X^{*}$ defined uniquely by

$$
(G(u), v)=a(u, v)
$$

for all $v$ in $X$. Then $G$ satisfies the conditions of Theorem 2 with $C=0$.

\section{BIBLIOGRAPHY}

1. F. E. Browder, Functional analysis and partial differential equations. I, Math. Ann. 138 (1959), 55-79. 
2. - On the solvability of non-linear functional equations, Duke Math. J. (to appear).

3. - Variational boundary value problems for quasi-linear elliptic equations of arbitrary order, Proc. Nat. Acad. Sci. U.S.A. (to appear).

4. - Variational boundary value problems for quasi-linear elliptic equations. II, Proc. Nat. Acad. Sci. U.S.A. 50 (1963), 31-37.

5. - Variational boundary value problems for quasilinear elliptic equations. III, Proc. Nat. Acad. Sci. U.S.A. (to appear).

6. P. D. Lax and A. N. Milgram, Parabolic equations, Annals of Mathematics Study No. 33, Princeton, N. J., 1954, pp. 167-190.

7. G. J. Minty, Monotone (non-linear) operators in Hilbert space, Duke Math. J. 29 (1962), 341-346.

8. M. M. Vainberg and R. I. Kachurovski, On the variational theory of non-linear operators and equations, Dokl. Akad. Nauk 129 (1959), 1199-1202.

9. M. I. Visik, Solution of a system of quasilinear equations having divergence form under periodic boundary conditions, Dokl. Akad. Nauk 137 (1961), 502-505.

10. - Boundary value problems for quasilinear strongly elliptic systems in divergence form, Dokl. Akad. Nauk. 138 (1961), 518-521.

11. - Simultaneous quasilinear elliptic equations with lower order terms, Dokl. Akad. Nauk 144 (1962), 13-16.

12. M. I. Visik and O. A. Ladyzenskaya, Boundary problems for partial differential equations and some classes of operator equations, Uspehi Mat. Nauk 111 (1956), 41-97.

INSTITUTE For ADVANCEd STUdy AND UNIVERSITY OF CHICAGo

\section{ERRATUM, VOLUME 48}

Arnold N. Lowan, Herbert E. Salzer and Abraham Hillman, $A$ table of coefficients for numerical differentiation, pp. 920-924.

$$
\text { Page } 924, A_{m, 8} \text { for } m=18, s=20 \text {, reads } \frac{117}{4} \text {, should read } \frac{177}{4} \text {. }
$$

